\title{
Student Understanding of Gravity in Introductory College Astronomy
}

\author{
Kathryn E. Williamson \\ Montana State University, Bozeman, Montana 59717 \\ Shannon Willoughby \\ Montana State University, Bozeman, Montana 59717 \\ Received: 08/31/11; Accepted: 02/3/12; Published: 03/12/12 \\ (c) 2012 The American Astronomical Society. All rights reserved.
}

\begin{abstract}
Twenty-four free-response questions were developed to explore introductory college astronomy students' understanding of gravity in a variety of contexts, including in and around Earth, throughout the solar system, and in hypothetical situations. Questions were separated into three questionnaires, each of which was given to a section of introductory college astronomy with 143, 137, and 32 respondents, respectively. Combined with 15 interviews, the exploratory, open-response format allowed themes to emerge naturally, with both documented and undocumented misconceptions observed. The breadth of questions allowed for descriptions of possible student mental frameworks, including alternative models and misapplication of the scientific model.
\end{abstract}

\section{INTRODUCTION}

The most recent and widely used pedagogy in cognitive science is the constructivist conceptual framework, which posits that an individual's personal ideas determine the way s/he processes external influences, and, as a result, the construction of knowledge is a personal phenomenon (Driver 1985, Fosnot 1996, Bransford, Brown, and Cocking 1999). However, these personal ideas are frequently shared by other students in the form of commonsense misconceptions or alternative theories about the physical world. Teachers and curriculum developers can help students to restructure their mental frameworks by understanding the interplay between students' deep-seated ideas and reasoning processes (Nussbaum and Novik 1982). Students typically have many preconceptions when confronting astronomy content, and one goal of Astronomy Education Research is to identify these preconceptions in the hope of improving astronomy education (Prather, Rudolph, and Brissenden 2009).

The concept of gravity is particularly important for teaching other astronomical concepts (Smith and Treagust 1988). Gravity is also a concept with which everyone has daily experience; therefore, it is an excellent starting point for investigating and reshaping misconceptions in astronomy. While the concept of gravity may be covered in only one or two lectures, its implications come up again and again throughout the semester. In conjunction with the typical learning goals for an introductory college astronomy class, an understanding of gravity can provide a stronger understanding of the causes of tides, Kepler's Laws and motions of bodies in space, differences between irregular-shaped asteroids and spherical-shaped planets, star formation, and Galileo's heliocentric model of the Solar System. An understanding of gravity adds perspective to our place in the universe, including the possibilities of spaceflight, the distances over which bodies in space interact, and the grand structure of galaxies.

A background review of seven Introductory Astronomy textbooks indicates that gravity is a concept that is covered in a typical astronomy course. Furthermore, students are expected to apply their understanding of gravity to other concepts throughout the course. Additionally, interviews with six Physics experts (see Note-1) 
indicate that gravity offers a good opportunity to think scientifically, a stated objective of many Astronomy teachers (Slater et al. 2001, Slater and Slater 2008) and a useful lifelong skill (Partridge and Greenstein 2003, Hobson 2008). The present study uses a grounded theory (Creswell 2007) framework within a constructivist pedagogy to explore student understanding of gravity in an introductory college astronomy course via openresponse questionnaires. The results show that college student understanding of gravity is lacking in many areas that are documented in younger students, but also new areas as they attempt to integrate classroom instruction with their previous knowledge. Providing exhaustive documentation of student misconceptions is necessary to move forward in the development of comprehensive assessment, curriculum improvement and, therefore, student learning (Prather 2009).

\section{REVIEW OF PREVIOUS RESEARCH}

\subsection{Student Understanding of Gravity at the K-12 Level}

There have been several studies of children's understanding of gravity. Nussbaum and Novak (1976) asserted "Five Notions" for children's understanding of the Earth's shape and gravity that guided much of the subsequent work. For a comprehensive compilation and review of the research on and recommendations for children's understanding of gravity, one may refer to Agan and Sneider (2004), Kavanagh and Sneider (2007a and 2007b), and Asghar and Libarkin (2010). The most common misconceptions held by very young children stem from a strong Earth-centered reference frame, including the idea that things can fall "down" off of the Earth. Middle school and high school students generally hold more sophisticated misconceptions, such as the idea that gravity needs a medium, such as air, to act through, that gravity is confounded with magnetism and rotation, and a general confusion among physical concepts such as weight, mass, and force.

To introduce the methods of the present study, we will concentrate our background review on research that utilizes student-supplied responses, with particular focus on studies done with middle school and high school students, as these investigations helped to guide the questions we asked students. For instance, Palmer (2001) asked sixth and tenth grade students to describe the force of gravity on a series of everyday objects that were depicted in various stages of motion near Earth's surface. The results suggest that student have a situational understanding of gravity, often predicting that the force of gravity is in the direction of an object's motion. For a brick buried under the ground, many students predicted that the brick would experience zero gravitational force from the Earth. After reviewing several student-supplied responses, Palmer developed seven concept categories to describe how students approach these situations, including categories in which the direction of the gravitational force is tied to the direction of motion of the object in question.

\subsection{Student Understanding of Gravity at the College Level}

Presumably children's and high-school students' misconceptions of gravity persist into college, and the few studies of student understanding of gravity at the college level support this supposition. The methods for researching college student understanding of gravity have been similar to those for researching children's understanding of gravity, typically using a few questions with diagrams and analyzing student-supplied responses. Piburn et al. (1988) used a series of diagrams with different sized planets at various distances from the Sun and asked physical science students which planet a rocket would be easiest to "take off" from, finding that many students viewed the presence of the Sun as a major factor in a planet's gravity. Dostal (2005) points out, however, that these questions may be a bit confusing, since real rockets do need to consider the relative position of the Sun. However, results of Piburn's study allude to deep-seated misconceptions, such as the idea that gravity emanates from the center of an object, and a planet's gravity is related to magnetism, temperature, composition, and distance from the Sun.

There have been a few studies of student understanding of gravity within Physics classrooms at the college level. These tend to emphasize physics terminology and laboratory experiments on Earth. Sharma et al. (2004) investigate Australian college physics students' conceptions of gravity by phenomenologically categorizing student responses to a question about why an astronaut in a spaceship has zero weight. Gunstone and White (1980 and 1981) asked first-year Physics students to compare the experimental results after dropping objects in class. As may be expected, students were reluctant to accept the scientific view, even when their classroom experiment contradicted with their prediction. Graham and Berry (1993) use a similar scenario by asking students to compare the motions of two balls dropped on the Earth versus on the Moon. Dostal's Master Thesis (2005) investigated college student understanding of gravity with emphasis on Physics force diagrams. The multiple- 
choice and open-ended questions required students to understand the principle of superposition, Newton's three laws, and proportionality within Newton's law of gravitation. While these studies include valuable exercises for Physics students grappling with terminology and notation, it is not readily applicable to Astronomy students, who often are not required to draw force diagrams, understand superposition, or articulate the differences between mass, weight, force, and acceleration.

To investigate a context outside of the typical Physics domain, Asghar and Libarkin (2010) adapted questions from studies that investigate children's understanding of gravity to research college students enrolled in an introductory geological sciences class. They found that college students' conceptions of gravity are generally more complex than children's, but misconceptions still abound. In particular, college students frequently confound gravity with magnetism, rotation, and atmospheric pressure, with only $12 \%$ of students holding the correct scientific conception without simultaneously holding alternatives.

The only study apart from this one that focuses on college student understanding of gravity in an introductory college astronomy course was done by Feeley (2007) for a Masters Thesis. He focused on scenarios on the Moon, including the motion of a pen, a feather, a piece of lead, and a helium-filled balloon released from near the surface. The responses suggested two primary models for student thought, which Feeley called the "air-gravity model" and the "threshold model." Students who used the air-gravity model reasoned that the Moon has no gravity because it has no air. Students who used the threshold model used the object's mass to determine its motion, with only heavy objects falling to the surface of the moon and lightweight objects floating away. Feeley also gave students information about Venus and then asked them to compare gravity on Venus to gravity on the Earth. Student responses to this separated into a "planetary rotation model," the "air-gravity model," and the Newtonian model.

Published studies on student understanding of gravity at the college level use only a few open-ended questions, or even just one, to explore student ideas about gravity. While these studies are useful in their own right, many misconceptions dealing with gravity are interdependent. The present study is a more comprehensive effort in that it asks students a variety of questions in different contexts and searches for emerging patterns. It is the most comprehensive study that we have found in the literature that is specifically tailored to focus on the concepts of gravity that are important in introductory astronomy courses.

\section{IMPORTANT CONCEPTS ABOUT GRAVITY TO FOCUS ON}

In compiling the questions for this project, we wanted to know which aspects of gravity are most important for introductory astronomy courses. With the foresight that the results would guide the development of a multiplechoice gravity concept assessment, we wanted a rather narrow focus. While Einstein's theory of relativity is often addressed in introductory astronomy courses, it is rarely emphasized. We deemed it unfair to ask questions that require an understanding of Einstein's picture, since it would be impossible to tell if the student was correctly applying Einstein's laws, or just had a misunderstanding of Newton's Laws. Thus, it was decided to only focus on aspects of Newtonian gravity.

To investigate the most basic and encompassing principles of Newtonian gravity, we sought general agreement among astronomy instructors, textbook coverage, and gravity experts. A review of seven textbooks indicates that gravity is generally presented starting with the historical progression, which closely parallels the progression of student conceptions about gravity (Kavanagh and Sneider 2007a and 2007b). Most textbooks then discuss the implications of falling, orbiting, and Newton's Laws in terms of planet formation, launching rockets, and tides. At this stage in the research, "a complete understanding of gravity for college introductory astronomy students" will be generally defined as "accurate knowledge and application of the basic underlying concepts associated with the Newtonian force of gravity."

We sought expert advice by interviewing five Physicists/Astrophysicists (see Note-2) and one Geological Scientist (see Note-3) and asking for feedback from AstroLearnr listserv members, of which 11 responded (see Note4). Personal communication with other Physics and Astronomy experts was also crucial (see Note-5). All agree that gravity is a foundational topic for astronomy and critical thinking in general. Discussions reveal that gravity has many, many facets. Some of the most important aspects that were brought up include the following:

- The universality of gravity: The same physical principles of gravity apply to every object in the universe.

- Influencing factors: The strength of gravity depends only on the mass of both objects and the distance between them (not size, density, rotation rate, composition, magnetism, etc.). 
- The scale and extent of gravity: A body's gravitational field falls off with distance but approaches zero asymptotically.

- Tidal forces: The Moon's gravity causes the tidal bulge of oceans on the side closest to and opposite the position of the moon.

- Gravity is an interaction: Gravity is a force of attraction between two masses and is equal for both objects based on Newton's third Law.

- Equivalence Principle: Gravitational mass and inertial mass are the same quantity.

- Action at a distance: Gravity is a noncontact force and cannot be blocked (for example, by another mass).

These aspects of gravity were further narrowed for the questionnaires to focus on those shown in the concept map in Figure 1. While the topic of tides is prevalent in most astronomy courses, it was eliminated because a thorough understanding requires higher levels of physics, such as gradients and multipole expansion of the gravitational field. It should be emphasized that this concept map is merely a guide; adhering too strictly to it could limit exploration of student conceptions. While it is convenient to separate different aspects of gravity, it is important to keep in mind that all are inherently related. Testing one will inevitably test another.

In compiling the questions to target the gravity concepts presented above, we were careful to separate as much as possible general aspects of "force" from the specific "gravitational force." Therefore, there is little to no emphasis on Newton's laws, as the Force Concept Inventory (FCI) is a more appropriate test of Newton's laws and, more importantly, it repeatedly shows that students have a poor understanding of forces (Hestenes, Wells, and Swackhamer 1992). It is for this reason that in formulating the gravity conceptual questions we were careful to emphasize aspects of the causes, effects, and extent of gravity, rather than general force concepts.

\section{METHOD}

\subsection{Free-Response Questionnaires}

We chose a ten-item free-response format as the best method to probe introductory astronomy student understanding of gravity. This serves three purposes: (1) to explore different representations of gravity in a variety of situations, (2) to allow for analysis via a constant comparative method, as discussed below, and (3) to gain information for distracter responses for a multiple-choice test that represent typical student answers in their typical language. In keeping with our goal of focusing on the causes, effects, and extent of gravity, the questions had little to no emphasis on equations. In order to reliably measure a student's mental model, we avoided questions where students needed to have heard the answer before, instead emphasizing questions whose answers can be deduced easily by a true understanding of gravity. These are generally different types of questions than those that are asked in textbooks at the end of the chapter. The questions explore the strength of gravity in a variety of environments and contexts—on the moon, in space, in freefall, on planets with orbiting bodies, etc.

In total, 30 questions were developed for the questionnaires, and these questions were divided evenly into three versions: A, B, and C (see Appendix A for a compilation of the questions that were used for this paper). Each section of introductory astronomy in the Fall semester of 2010 was given 30 minutes to complete a different version of the questionnaire. Half of the questions in each version of the questionnaire were composed in text only, and the other half provided a figure of the situation described in the question.

\subsection{Population}

All questionnaires were given to students enrolled in an introductory Astronomy course at Montana State University during the Fall 2010 Semester approximately 3 weeks after the lesson about gravity. There were three sections, two

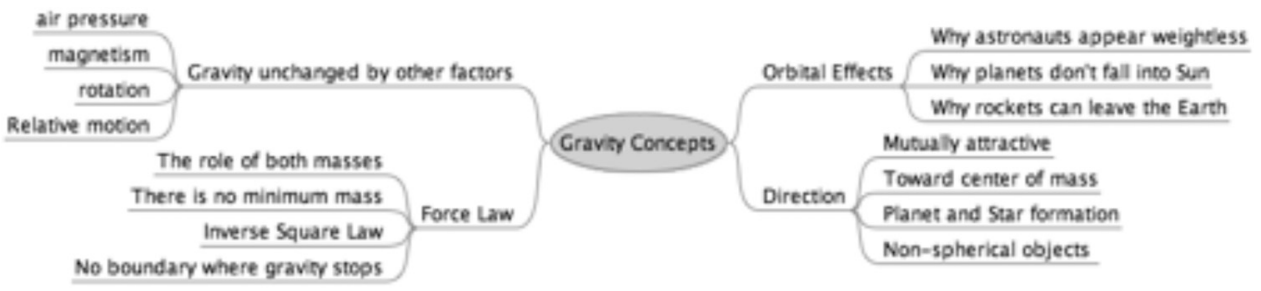

Figure 1. A guiding concept map used to determine the types of questions that were given to students in the form of openended questionnaires. 
of which were taught by one of the authors. Section 1 included 190 registered students, 143 of which participated in Version A of the gravity survey, giving a response rate of 75\%. Section 2 included 191 registered students, 137 of which participated in Version B of the gravity survey, giving a response rate of $72 \%$. And, Section 3 included 54 registered students, 32 of which participated in Version C of the gravity survey, giving a response rate of $59 \%$. Because responses to Version C were low in number, interpretation of student responses to this version should be viewed with caution. Students who typically register for this course are nonscience majors wanting to fulfill their science requirement. Therefore, this may be the only science course these students take in college (Partridge and Greenstein 2003). Approximately 38\% of the students registered for the course were female, but the gender ratio for those students who participated in the gravity questionnaires is unknown.

\subsection{Constant Comparative Approach}

In analyzing student responses, we followed the model presented by Creswell (2007) for a grounded theory constant comparative research approach. This approach has been used in many studies on student conceptual understanding (Bailey 2006, Sharma et al. 2004, Asghar and Libarkin 2010), and it has proven to be a reliable method. The central idea to constant comparative research is an open-coding format where the researcher collects data and allows categories to emerge naturally until the information gained is saturated. In constructing categories of student responses to each question, we took an approach similar to what Sharma et al. (2004) refer to as a phenomenographic analysis, which de-emphasizes correctness in favor of probing for a complete description of student ideas.

One author compiled student responses by typing each response into a single document and giving each response a number tag so that individual cases could be tracked. In an exploratory fashion, the student responses were read, reread, and rearranged to determine the emergent themes for each question. Once the general themes, or categories, were determined, each author followed the constant comparative method by separately coding the numbered student responses into the determined categories. To illustrate how these codings were compiled and analyzed, consider an example of ten student responses (\#1 - \#10) to a question that has two conceptual categories, 'a' and 'b.' Table 1 shows how each rater in this example coded each student response. The raters agreed that four responses $(\mathrm{N}=4)$ fit in Category ' $a$ ' and three responses $(\mathrm{N}=3)$ fit in Category ' $b$.' The raters did not agree on the categorization of two responses (\#5 and \#9). For example, Rater 1 coded response \#5 in Category ' $b$ ' while Rater 2 coded it in Category 'a.' Also note that both raters deemed response \#2 inapplicable to the question.

To measure the degree to which our two codings of student responses about gravity agreed, we used a matrix similar to Table 1 to calculate the Cohen's Kappa inter-rater reliability statistic, $\kappa$, for each question. Inter-rater reliabilities greater than 0.80 are generally accepted as good agreement (Landis and Koch 1977). If the categorizations for any question resulted in a $\kappa$ below 0.80 , discussions and clarifications were made in an iterative process until acceptable agreement was reached, but no explicit information was exchanged as to which responses each researcher placed in each category.

\subsection{Follow-Up Interviews}

One of the authors conducted follow-up, audio-recorded interviews to establish credibility of the questionnaires and to gain insight into the complexities of student reasoning. As an incentive, students were offered the chance to win one of five \$20 Target gift cards. Fifteen students volunteered for interviews - this included five students who completed version A, nine students who completed version B, and one student who completed version C. All interviews were completed at the end of the semester, between 4our and 7 weeks after the gravity questionnaires were given in class.

The interview format was exploratory. Student ID number was used to match interviewees with their questionnaires completed in class. Students read the question and their written response aloud and were then

Table 1. An example of the codings of student responses by two raters.

\begin{tabular}{|c|c|c|c|c|}
\hline & & & Rater 1 & \\
\hline \multirow{4}{*}{ Rater 2} & & Category 'a' & Category 'b' & Not applicable \\
\hline & Category 'a' & $\# 1, \# 3, \# 6, \# 10$ & \#5 & \\
\hline & Category 'b' & \#9 & $\# 4, \# 7, \# 8$ & \\
\hline & Not applicable & & & \#2 \\
\hline
\end{tabular}


asked to clarify or expound as necessary. The interviewer was careful to ask probing, but not leading, questions. Some questions required little probing; however, some questions were explored a great length to test the strength of a student's mental framework. Interviews typically lasted 20-30 minutes.

\section{RESULTS}

\subsection{Questionnaire Results}

Appendix A provides a comprehensive summary of questionnaire results. While the questionnaires contained a total of 30 questions, results from only 23 questions are presented. The other seven questions were generally misunderstood by students or provided no new, previously undocumented, information. Each question is displayed with accompanying conceptual categories in the first column and total number, $\mathrm{N}$, of responses that fell within each category according to both raters in the second column (as presented in Section 4.3). Note that the number $\mathrm{N}$ does not include student responses that were placed in different categories according to each rater, or incoherent or inapplicable student responses, as these responses were not useful in understanding students' understanding and reasoning on questions. Therefore, the sum of the $\mathrm{N}$ values across categories does not match the population outlined in Section 4.2. The third column in Appendix A expresses $\mathrm{N}$ for each category as a percentage of total respondents, i.e., $\mathrm{N}$ was divided by 143 for responses from Version A, 137 for responses from Version B, and 32 for responses from Version C. Finally, the fourth column provides the interrater reliability statistic, $\kappa$.

\subsection{Interview Results}

Interviews can be categorized as "Consistent," meaning that a student appeared confident in his or her responses and consistently applied similar reasoning strategies, or "Inconsistent," meaning that a student was very unsure of his/her responses and changed reasoning throughout the interview. Appendix B summarizes student interview results, including examples of possible working mental models (explained in Section 6).

Question 1A proved to be an excellent starting place to probe students' mental models. The majority of students answered that astronauts appear to float in their spacecraft because there is no gravity in space. These students were then asked related questions to bring up contradictions in their reasoning. This is demonstrated in the following transcript between a student (S) and interviewer (I):

S: "Why do astronauts appear to float in their spacecraft?" My response is "there is no gravity in space."

I: Okay, and do you still agree with that?

S: Yes, I do.

I: Okay, and what do you define as "space"?

S: Anything outside of our gravitational pull.

I: So is there a boundary where something is in our gravitational pull and then not?

S: I think it gradually decreases from Earth's surface to nothing at some elevation above earth.

I: Astronauts orbit Earth just outside our atmosphere, so I guess I'm asking if it [gravity] decreases fast enough to get to be zero just outside our atmosphere.

S: Yes.

I: So then is the gravity from the Earth zero at the Moon? Does the Moon feel Earth's gravity at its location?

S: No, the Moon has its own gravity.

I: But the Moon orbits the earth, right?

S: True.

I: So why does it orbit the earth?

$\mathrm{S}$ : Because of the gravitational pull between the two masses.

I: Okay, so it seems that you're saying that the Moon does feel the Earth's gravity.

S: Yes.

I: So why can the Moon feel Earth's gravity, but the space station can't?

$\mathrm{S}$ : Because the space station isn't a big enough mass.

This example reveals how misconceptions are often intertwined-this student traded in one misconception for another. In answering that there is no gravity in space, the student may appear to conform to the common misconception that gravity needs air to act through. However, thinking about Earth's effect on the Moon helped this student to realize that Earth's atmosphere does not affect gravity. Therefore, a more appropriate category for this misconception is that gravity only applies to heavy objects. 


\section{STUDENT ALTERNATIVE MODELS OF GRAVITY}

The compilation of student responses to the breadth of questions allowed for a description of emergent thought patterns, which suggest potential student alternative "mental models" as described by diSessa (1993) and Redish (1994). In each subsections below, we present each emergent mental model in the general sense as in Bao and Redish (2006) — as "a robust and coherent knowledge element or strongly associated set of knowledge elements." In reading each subsection, it is important to keep in mind that each mental model is not independent—student misconceptions about gravity are generally inextricably linked. We would also like to emphasize that these mental models are by no means a complete description of all of student reasoning about gravity.

\subsection{The Boundary Model}

Perhaps the most prominent emergent theme is what we call the "boundary model." A boundary could be the surface of a planet, the edge of the atmosphere, or even an orbit. It may represent a casing that encloses a planet's gravity, or a "check point" where gravity either disappears or diminishes. With the boundary model, we can explore several student responses.

A combination of Questions A2, B10, and A1 illustrates the boundary idea in very specific regions of Earthinside the Earth, above the surface, and beyond the atmosphere, respectively. For Question A2 many students $(\mathrm{N}=37,25.9 \%)$ reason that the strength of gravity would not change if one traveled down a tunnel to the center of the Earth. The most common explanation for this is that Earth's gravity is constant throughout, because the Earth is one body ( $\mathrm{N}=33,23.1 \%)$. In this case it is the Earth's surface that acts as a boundary, inside of which Earth's gravity is constant. However, Question B10 illustrates that most students $(\mathrm{N}=71$, $51.9 \%$ ) understand that Earth's gravity diminishes with distance from the surface. Considering further distances from the surface of Earth, the majority of respondents to Question C17 ( $N=15,46.9 \%)$ assert that one simply must leave the Earth (and its atmosphere) to feel zero gravitational force from Earth. This is a welldocument misconception (Watts 1982, Asghar and Libarkin 2010) and is supported by typical responses to Question A1 that assert astronauts appear to float in their spacecraft because there is no gravity in space $(\mathrm{N}=$ $72,50.3 \%)$. In the "boundary" concept, there are many ways this could make sense: the atmosphere is the casing that holds gravity inside, or the extent of the atmosphere is simply an indicator of the presence of gravity. The latter explanation is clearly articulated in many student responses to Questions A1 and A3. An interviewee discussing his response to $\mathrm{A} 1$ asserts:

S. Astronauts are able to float in their spacecraft because they've broken out of the atmosphere... once they've gotten past the point where earth is able to sustain any kind of atmosphere, then that's where gravity is weak enough for it [floating] to happen. I don't think it's a correlation in any meaningful sense. It's not like cause and effect or anything.

I: So it's not like the atmosphere is causing gravity?

$\mathrm{S}$ : Where the atmosphere is, gravity is strong enough for there to be gravity.

I: Atmosphere is more of an indicator of where gravity is working?

S: Yeah, that would be how it works. Yeah.

Combining these typical responses, we arrive at several possible student models for discontinuities, or boundaries, in Earth's gravitational influence. Variations of the boundary idea in and around Earth are presented in Figure 2.

In the present study, the misconception that there is no gravity in space was one of the most prevalent. As previously mentioned, this surfaced in student responses to Question A1, but it was also prevalent in responses to Question B10, where 14.6\% ( $\mathrm{N}=20)$ of respondents reasoned that the strength of the Earth's gravity on top of Mt. Everest is weaker than at sea level because Mt. Everest is closer to space. In Question B12, a few students asserted that the Apollo spacecraft experienced no gravity because it was in space. And, in Question C23, $12.5 \%$ $(\mathrm{N}=4)$ of respondents asserted that two astronauts in space do not experience a gravitational force because there is not gravity in space.

Within the context of the boundary model, it may be reasonable to assume that anytime a student asserts that there is no gravity in space, s/he is working from the idea that "space" is always outside the boundary, whether it be at the surface of a planet or past its atmosphere. As indicated in the interview transcript presented in Section 
6.2, the boundary may be different for different orbiting objects, extending out farther for more massive objects. The boundary may not define the edge of "space" at all, but rather the conditions for orbiting to occur, inside of which an object falls to a planet, and outside of which an object flies off into space. Responses to Question B8 use the boundary concept in terms of orbits to explain why the planets do not fall into the Sun: "Gravity is pulling the planets towards then Sun but the gravity is not strong enough to actually pull them in," and, "They are so far away and the Sun doesn't have enough gravity," for example. In this case, the "boundary" divides regions where the Sun's gravity pulls objects in and where it simply holds them in orbit. While this may not be completely incorrect, as planets are in equilibrium, it is clear that students do not understand why the planets are in equilibrium.

\subsection{The Orbital Indicator Model}

An unexpected misconception that was uncovered is that the objects that orbit a planet can act as indicators of its surface gravity. This was strikingly revealed by the majority of student responses to Question B14. This question showed equally sized planets with orbiting satellites or moons at different orbital radii. Categories "a" and "b" show how the "orbiting indicators" concept is generally applied in two opposing ways: (a) A planet's gravity must be stronger to reach out and hold objects that are far away, or (b) A planet's gravity must be stronger to pull objects close to it. A typical student response falling in Category "a" is as follows:

"[Planet] A: not much gravity, B and C [have the] same gravity, D [has the] most gravity because it can have a satellite [at the] furthest [distance]. E, F, and G have tides. G has the strongest gravity [because its] moon is furthest away, but $\mathrm{E}$ and $\mathrm{F}$ both have relatively strong gravity. F might not have tides b/c of [its] two moons."

This comment contains several interesting concepts. First, in claiming that planet A's gravity is minimal, the student indicates A's gravity is not strong enough to hold any objects in orbit. Second, claiming that B and C have the same gravity and less than planet $\mathrm{D}$, the student assumes that the gravity of planets B and C must not reach as far, and must, therefore, be weaker than the gravity of planet $\mathrm{D}$. The student applies this same reasoning for planets E, F, and G. Because the student separated their predictions for the strength of gravity according to planets with artificial satellites and those with moons, s/he possibly expects planet G's gravity to be stronger since it is able to hold a heavier object, such as a moon, in orbit.

A typical response for Category "b" is as follows: "Probably no surface gravity on A b/c nothing is orbiting it, and a little more surface gravity on B-D b/c a machine is able to stay in orbit, and E-G has the strongest, most surface gravity b/c a moon is able to orbit. E-G probably have tides b/c of the moons." Like the previous student who fell in Category "a," this student reasons that Planet A must have weak (in fact, zero!) surface gravity because there is nothing orbiting it. To the student this logically implies that Planet A's gravity is simply not strong enough to hold objects in orbit. Unlike the previous student, however, this student claims that Planets B and C have more gravity than Planet D, and, similarly, Planets E and F have

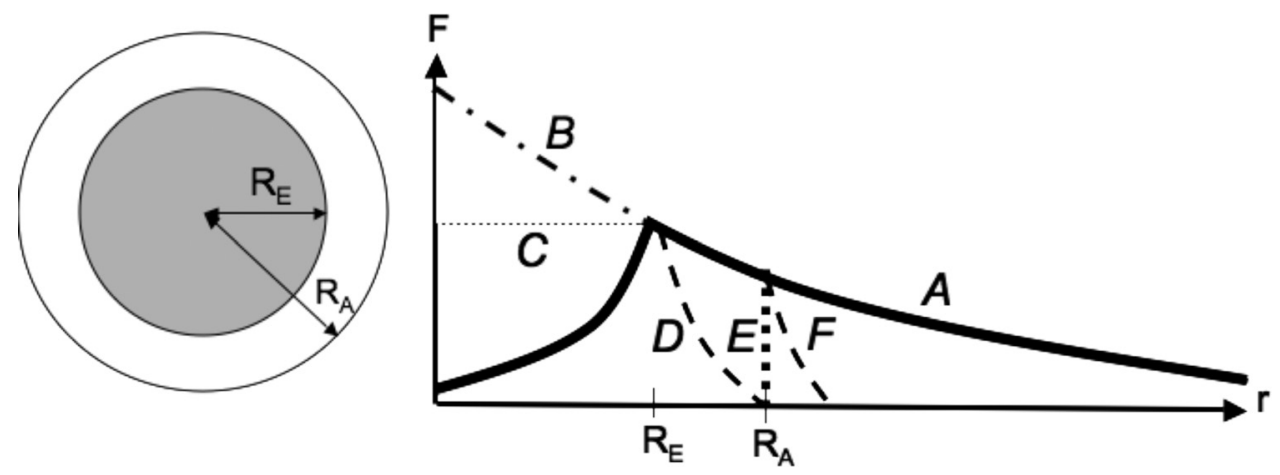

Figure 2. Variations of the boundary model for the strength of the gravitational field, F, as a function of distance from Earth center, r. (A) The correct scientific model, (B) the misconception that gravity is strongest at the center of the Earth, (C) the misconception that Earth's gravity is constant throughout because it is a single mass, (D) the misconception that Earth's gravity drops to zero at the edge of the atmosphere, (E) the misconception that the atmosphere encases the Earth's gravity, and (F) the misconception that gravity very quickly diminishes to zero outside the atmosphere. Students may hold any combination of these models. 
more gravity than Planet G. The reasoning here is that a planet's gravity must be stronger to pull an object closer to it.

This misconception comes up again in Question C21. Instead of satellites or moons orbiting a planet, different ring systems are depicted. Again, Categories "a" and "b" represent the two opposing applications of the "orbiting indicator" misconception. Analogous to Question B14 Category "a," students in Category "a” of Question C21 predict that the gravity on a planet with more rings and thicker rings must be stronger to hold so much material around the planet. For example, one student asserts, "If E can keep such far-away rings in check, it is exerting more force than B is on its closer-in rings."

Conversely, Category "b" of Question C21 indicates that planets with less material in orbit have a stronger gravity. A student response in this category is: "The planet with more mass does not have rings, and [for planets with] less mass the planet carries the surrounding objects around it in orbit instead of having it become part of the planet." Presumably the student is referring to "gravity" when s/he refers to "mass." This implies that planets with strong gravity will cause all orbiting bodies to spiral inward and impact the planet. If a planet's gravity is weak, then it may allow some objects to steadily orbit. Again, this is analogous to Question B14 Category "b", where planets with stronger gravity are able to hold orbiting bodies at smaller radii.

\subsection{The Mixing of Forces Model}

Questionnaire results overwhelmingly support the well-documented (Piburn 1988, Smith 1988, Chandler 1991, Graham and Berry 1993, Sharma et al. 2004, Feeley 2007, Asghar 2010) misconception that gravity is confounded with magnetism, rotation, and atmospheric pressure. Question A3 explicitly asks students how gravity is related to atmosphere (if at all), Question A7 explicitly asks how magnetism affects gravity (if at all), and Question B13 explicitly asks how rotation affects gravity (if at all). Perhaps it is understandable for these questions that the majority of students assert that the atmosphere affects the strength of gravity and strong magnetic fields correlate with strong gravity. However, students readily incorporate magnetism, rotation, and atmospheric pressure into their responses to questions that do not address these forces explicitly. All three of these forces are popular responses in Question B9, which asks, "Besides gravity, are there any other forces that hold us to the surface of the Earth?" While it is encouraging that the majority $(56.2 \%, \mathrm{~N}=77)$ of students understand that gravity is the only force holding objects on Earth, 12.4\% ( $\mathrm{N}=17)$ of students believe that atmospheric forces contribute, $6.6 \%(\mathrm{~N}=9)$ believe rotation and orbital dynamics contribute, and $2.2 \%(\mathrm{~N}=3)$ believe magnetic forces contribute. A few students who answered Question A5 even reasoned that people at different latitudes on Earth would experience different forces of gravity because of variations in the magnetic field $(2.8 \%, \mathrm{~N}=4)$ or variations in rotational speed $(4.2 \%, \mathrm{~N}=6)$. A few students refer to these forces again in other questions such as A2 and C18.

Responses to Question B8, "Why don't the planets fall into the Sun?" illustrate this trend, with 5.1\% (N = 7) of students claiming that rotation of the planets and/or Sun counteracts the inward pull of gravity and another $5.1 \%(\mathrm{~N}=7)$ claiming that repelling magnetic forces counteract the attractive gravitational forces. Responses in category "g" of Question B8 indicate an interesting misconception that could potentially be tied to magnetism. Students in this category reason that planets do not fall into the Sun because, "The planets have their own gravity that repels them from being drug in," or, "They (the planets and the Sun) are pulling against each other." The comment, "Gravity is pushing away from the Sun," indicates that some student may believe that gravity is analogous to magnetism, at times attracting and at other times repelling.

We propose a "mixing of forces model" to describe the types of student responses we observed. Magnetism, rotation, and air pressure seem to be important factors in understanding gravity for some students. The mixing of forces model perhaps could also be used conjunction with the boundary concept presented in Section 6.1. To many students, these three factors can modify the reach, or extent, of gravity. Perhaps this can be tied to misconceptions about force and superposition. Perhaps students are drawing on everyday experiences where they (or objects they hold) "feel" lighter or heavier, and they attribute changes in apparent weight to changes in gravity. Since all three versions of the questionnaires contained questions that explicitly address external factors, it is possible that students were cued by questions throughout the survey and they displayed these misconceptions more often than they would have without a prompt. Future research will need to be done to 
determine exactly how prevalent the mixing of forces model is and how students incorporate it into their mental frameworks.

\subsection{Disparate Misconceptions}

A few unexpected misconceptions surfaced from the student-supplied responses that do not seem to relate to a specific "mental model" of gravity - they appear to stand on their own as disparate topics. The most surprising misconception observed is the belief that South is "down." This has been documented in young children (Nussbaum and Novak 1976) but certainly not college students. However, 5.6\% (N=8) of the student responses to Question A5 indicate that a person at the North Pole of Earth experiences a stronger gravitational force than a person at the South Pole. Examples of student responses in this category include, "Person A is at the top and wouldn't fall off," and, "Person A is at a position where gravity is pulling them directly down." None of the students who fell in this category volunteered to be interviewed.

Another interesting misconception is the idea that gravity is a force that pulls an orbiting body along in its orbit, or that gravity establishes an orbit, which then "holds" the object. Responses in Category "c" of Question B8 seem to indicate that this is how some students understand orbiting, reasoning that the planets do not fall into the Sun "because the orbit has been established," or, "because their orbits keep them in place." One student even explicitly stated, "When planets are in orbit, the force of gravity between the two bodies is not a direct pull towards each other." Perhaps this is loosely tied to the mixing of forces model, where the force of gravity is intertwined with rotation or centripetal forces, or perhaps it is just an extension of the "orbital indicator model."

One more interesting misconception is that gravity can be blocked or stifled, such as when the two objects are not in view of each other. This was most prevalent in responses in Category "c" of Question B12. Students in this category reasoned that when the Apollo spacecraft was behind the Moon, "the Moon was in the way of Earth's gravity," or the Moon "interfered with" or "interrupted" the Earth's gravity. One student stated "Earth had no effect on the spacecraft once behind the moon. It would take effect again once it had view of Earth." This misconception could potentially relate to that of Category " $b$ " in the same question, where some students appear to believe that an object can only experience the gravity of one object at a time. In this case, when the spacecraft is near the Moon, some students wrote, "the Moon's gravity was no longer competing with the Earth's," or, the spacecraft "was in the Moon's gravity, not experiencing the Earth's gravity at all." Again, this could potentially be tied to the "Mixing of Forces model," but it seems more related to a misunderstanding of superposition. Perhaps these students are too literal in their interpretation of gravity as a force of attraction between two objects.

\section{STUDENT MISAPPLICATION OF THE SCIENTIFIC MODEL}

As Section 6 illustrates, students can have diverse mental landscapes of alternative models about gravity. However, this does not imply that they cannot also hold misconceptions related to the correct scientific model. In Sections 7.1-7.4, we discuss three main ways in which student responses indicated a failure to use the scientific model correctly: misapplication of mass, misapplication of distance, and misunderstanding what causes or creates gravity.

\subsection{Misapplication of Mass}

Students misapply the factor of mass in determining the force of gravity in a couple ways: (1) assuming that only heavy objects can gravitationally interact and (2) confusing mass and density.

\subsubsection{Only Heavy Objects can Gravitationally Interact}

Responses to Question A4 show that 35\% ( $=50)$ of students believe that there is a nonzero minimum mass requirement for an object to gravitationally interact with other objects. Of these $35 \%, 3.5 \%(\mathrm{~N}=5)$ of students come close to the scientific understanding by asserting that, for example, an object must be "probably a pound or more." An additional 7.0\% ( $\mathrm{N}=10)$ assert that it must be planet or moon sized. More ambiguously, $18.9 \%$ 
( $\mathrm{N}=27$ ) simply state: "very, very heavy." This misconception surfaces again in Question C23, which shows two astronauts in space far away from other objects. Only $37.5 \%(\mathrm{~N}=12)$ of respondents correctly assert that the two astronauts experience a force of gravity from each other. However, $18.8 \%(N=6)$ of respondents say that the astronauts feel a force of gravity from distant objects. While this group of students appears to understand that the strength of gravity diminishes to zero only over infinite distances, they do not appear to understand that any mass can interact gravitationally with other objects. Perhaps this is a more general application of the "threshold model" presented by Feeley (2007), in which students reason that a pen released near the surface of the Moon would not be heavy enough to fall.

\subsubsection{Mass Versus Density}

It appears that students have an intuitive understanding that more "stuff" means more gravity. This appears to be a phenomenological primitive, or "p-prim" (diSessa 1993), that students unquestioningly apply in their reasoning about gravity. It often serves them well, since more mass really does mean more gravity. However, many students assume that higher density always implies higher mass, without taking size into account. Because the density of a planet indirectly provides information about its mass (and hence its gravity), this misconception is particularly difficult to untangle. Some students go so far as to consider density as the primary factor and mass as the secondary factor that determines the strength of gravity, as demonstrated in the following student's interview response to Question A4, which asks how heavy something has to be to have gravity:

"I think it's gotta be pretty heavy... Something that's incredibly massive and incredibly light wouldn't necessarily be as much gravity as something that's equally massive but far denser. So I think it has something to do with size, but more to do with density and mass and such, because if the Sun was far larger it wouldn't be able to attract planets to it. Like I learned that when the Sun becomes a red giant it's going to push all the planets out, and I figure that's why_-because it's so much less dense as a red giant than something like a yellow star like it is right now."

This student is making a genuine effort to think scientifically by linking his reasoning with other information that he learned in class. He applies this same mental model throughout his interview responses. For Question A5, he reasons that a person at the equator would experience stronger gravity because the Earth bulges, and, "assuming the Earth's density is constant," mass becomes the determining factor, and, "there is more stuff between the person and the center of the Earth." Even though this student has misconceptions about the relationship of density, mass, and gravity, he works from a consistent mental model.

Alternatively, one must not discount the possibility that students are working from the correct mental model and simply using the wrong terminology, i.e., maybe students understand gravity, but they do not understand the difference between mass and density. Question A2 uniquely addresses this subtlety by asking students to imagine a thin tunnel that reaches to the center of the Earth. Some students reason that gravity would increase because "the center of the Earth has a greater mass," or, "the densest matter of Earth is in the core." Of course, the center of Earth cannot contain more mass than the entirety of the Earth, so it is reasonable to assume these students mean "density" when they say "mass."

\subsection{Misapplication of Distance}

Many students understand that distance between two objects is an important factor in gravity; however, misconceptions occur in how this is applied. For instance, many students become attached to the word "radius." Scientists often use the variable $r$ for "distance," but students may interpret this too literally and deduce that $r$ stands for "radius," and a smaller planet has stronger gravity because it has a smaller radius. This is exemplified in Category " $h$ " of Question A6, with a typical student response: "A is strongest, D is weakest. Masses will be the same but the radiuses [sic] change. The smaller the radius the greater the [gravitational] force." This misconception crops up again in Category “e” of Question B11. One student reasoned that Pluto's gravity would be stronger than Earth's "because the surface is closer to the core."

An alternative misapplication of the distance variable is mistaking distance as the distance from the surface of a planet. A quote from Category " $\mathrm{e}$ " of Question A6 is an example of a student considering the distance from the surface of the planet: "The darker the planet the more dense. Gravity is affected by mass and distance. In the darker shaded planets they will experience more gravity. In the lighter shaded planets they are closer to the planet causing greater gravity." 


\subsection{Misunderstanding What Causes or Creates Gravity}

Many students do no understand where gravity comes from. One interviewee explicitly states, "I don't know what creates gravity. I just know it exists." This is extremely detrimental to understanding how gravity works and how to use the force law, in particular. Despite understanding that mass is an important factor in determining gravity, many students fail to make the connection that the interaction of two masses is in fact the cause of the gravitational force. Because examples and problems in class primarily involve spherical bodies, the center of the object is frequently referenced and students begin to think that the center is somehow different or more special than the rest of the mass. In fact, some students reason that the geometric center of an object is (1) where gravity originates, or (2) the location to which gravity pulls everything.

Question A2 illustrates that many students believe the center of Earth to be somehow different gravitationally than the rest of Earth. 23.1\% ( $=33)$ of students reasoned that gravity would be stronger at the center of Earth than at the surface, with an additional $4.9 \%(\mathrm{~N}=7)$ implying that this is because the center is the source of gravity, providing comments such as "we are closer to the source of the Earth's gravity, where gravity originates," and "you are getting closer to the mass exerting the gravitational force." The former comment may provide insight into another facet of this misconception-it is not necessarily the center point where gravity originates, but a central region. Some students draw on their knowledge that the core of the Earth is compositionally different than the crust. It is denser, and a student who believes that density, rather than mass, determines gravity would reasonably conclude the center exerts a stronger gravitational force than the crust. Also, the core of Earth is primarily iron, which could potentially factor into a student's mental model that includes magnetism. And finally, a student who believes pressure (atmospheric or otherwise) to be associated with gravity would logically conclude that the center of Earth exerts a stronger gravitational force than the rest of the Earth.

Many responses to Question C22 further demonstrate the misconception that gravity originates from the center of an object. Depicting a planet whose center of mass is offset from its geometric center unambiguously untangles student conceptions. While $34.4 \%(\mathrm{~N}=11)$ of respondents correctly drew an arrow representing the gravitational force toward the center of mass, $21.9 \%(\mathrm{~N}=7)$ drew an arrow to the geometric center. In the latter case, one student adds that, although gravity still points to the geometric center, "it may be weaker than normal." Again, students may understand that mass is a factor in a planet's gravity, but they do not understand its role exactly. Alternatively, a few students $(9.4 \%, \mathrm{~N}=3)$ appear to believe gravity to be caused by a lack of mass, as these students drew arrows pointing into the hole of the planet. Since version C had so few respondents, we can only speculate as to students' reasoning in this process. However, arrows to the hole may allude to the misconception that gravity is measured by its effects on other bodies-a person could fall into the hole but would merely remain on the surface at the side without a hole.

Student responses to Question A2 unveil the subtle interplay between mass and distance. In considering how gravity changes upon traveling down a thin tunnel to the center of Earth, some students who understand the proper relationship between gravity and distance (i.e., they wrote Newton's law of gravitation) reason that gravity increases because "the radius is smaller." Perhaps these students assumed that, since the Earth's mass is constant, they could consider only distance. They ignore how the relative distribution of mass affects gravity at a point inside the Earth. While a truly proper explanation for the decrease of gravity requires a Gaussian approach, students who answered correctly $(3.5 \%, \mathrm{~N}=5)$ reasoned that gravity would decrease because, "Some of the mass of the earth would be behind you and pulling in the opposite direction of the rest of the earth," and there is "less mass between you and the center of the body of mass."

\section{DISCUSSION}

\subsection{Model Mixing}

The misconceptions presented above are not necessarily mutually exclusive and may contribute to each other. For example, a student who asserts that the Earth's magnetic field and/or rotation can push out and extend the Earth's gravitational field may simultaneously hold the boundary model misconception and the orbital indicator misconception. Even students who correctly apply the scientific model may also simultaneously reason with alternative models. We speculate that students that mix seemingly contradictory models are operating from what Bao and Redish (2006) call a "mixed model state," within which individual models are triggered by situational cues. While seemingly contradictory to the expert, students might use one model in a certain situation and a different model in a different situation. We 
found that, in general, only when pushed with questions (such as in interviews) do students begin to question their framework and modify it accordingly.

Despite the multitude of misconceptions about gravity that students can possess, the majority of responses to most of the questions fell within a category that fit with the correct scientific model of Newtonian gravity. However, unlike Piburn (1988), we do not conclude that this means that students mostly have a reasonable understanding of gravity. Section 7 elucidates the many ways that students get lost in their attempts to reconcile Newton's law of gravitation with their intuition. They learn that mass and distance are the primary factors that determine the strength of gravity, but in many cases they do not know how to apply these factors: Is the "distance" the radius of a planet or the distance of an orbiting body from the surface of a planet? Are mass and distance the only factors that determine gravity? How does mass relate to density? It was rare for a single student to possess the correct scientific model without simultaneously relying on an alternative model to answer certain questions. This is in agreement with the results found by Asghar and Libarkin (2010). We suspect that students are trying to "assimilate," rather than "accommodate" in Piagetian terms (Vosniadou and Brewer 1987), what they are taught about gravity with their previous knowledge.

When intuition takes precedence, students often deduce the strength of gravity based on its effects, or what we would feel like in that situation. They have a hard time distinguishing between apparent weight and gravitational force. The orbital indicator model may be part of a broader mental framework that gravity can be understood in terms of its effect on other objects. For example, student responses that fell in Category ' $b$ ' of Question B14 indicate that a small satellite orbiting Earth would experience a stronger gravitational force than a larger satellite, because "it is easier to move." Furthermore, responses in Category ' $d$ ' of Question A4 $(5.6 \%, \mathrm{~N}=8)$ indicate that some students believe a planet's gravity to be defined only in terms of its effects on other objects. Responses in this category describe how heavy or light something must be to create its own gravitational field with such responses as: "Heavier than all other objects near it," "Depends upon how big other objects are," and "It would depend on where it is placed. If the object is on earth it would have no gravitational field, but if it were in space it would have one." Determination of gravity based on its effects has been documented by Noce (1988) in children and by Asghar and Libarkin (2010) in college geology students. Perhaps this misconception relates to misconceptions about forces in general. Students think in terms of the effort it would take to move an object, with the bigger, stronger objects being "in control over the smaller objects."

\subsection{Gravity is Confusing}

One other noteworthy trend is that many students were very unsure of their answers to questionnaires. This was obvious in several written responses where students explicitly state, "Not sure," or, "I have no clue." However, it was especially prevalent in the interviews. Five of the 15 interviewees (33\%) were categorized as "Inconsistent" in their reasoning (see Appendix B). Even those who were categorized as "Consistent" did not seem entirely confident in their answers. As they reviewed their written responses, several rambled and wavered back and forth before making a decision. Interviewee \#4 felt the need to express: “... oh, and I worded everything so I sound confident, but I'm not super confident."

\subsection{Questionnaire Limitations}

For a body in orbit around a planet, the gravitational force law requires the mass of both the body and the planet; however, in calculating the acceleration due to gravity, only the mass of the planet needs to be considered. Understanding this distinction may not be necessary for introductory astronomy students, so in many questions we simply refer to "gravity," rather than specifying the "force," "acceleration," or "field." In hindsight, this may not have been entirely appropriate, as some students misinterpreted questions. Question B15 highlights this ambiguity_two common response categories were that 'a': Larger, more massive satellites experience a stronger force of gravity, and 'd': Gravity pulls on all objects equally. Category ' $a$ ' is the correct response for gravitational force, and Category ' $d$ ' is the correct response to gravitational acceleration. Question A4 shows similar confusion: 5.6\% $(\mathrm{N}=8)$ of respondents reason that an object only has gravity if it has an effect on another object. While it is true that gravitational force requires two objects, the concept of a field allows one to think about a single object's gravity. It is impossible to distinguish what the student meant or if they have misconceptions about these topics at all. In future multiple-choice versions that incorporate these questions, we will be more specific with terminology to eliminate ambiguity. 


\section{CONCLUSIONS}

\subsection{Summary}

A students' understanding of gravity is closely related to his/her understanding of other physical concepts, such as air pressure, the presence of an atmosphere, momentum, kinematics, and magnetism. For this reason, gravity can serve as a proxy for student's understanding of many general physical concepts. Gravity is a concept that everyone has heard of and has daily experience with, so it is an excellent starting point for studying and reshaping students' mental models. In the Constructivist framework, defining the preconceptions and misconceptions that students work from is a critical first step in helping them restructure their mental models.

The present study used exploratory, open-response format questionnaires to explore student misconceptions about gravity, which allowed themes to emerge naturally in students' typical language. This is the first comprehensive study tailored specifically to introductory college astronomy students' understanding of gravity. A variety of contexts were explored, including the strength of gravity in and around Earth, in terms of the solar system, and in hypothetical situations. Fifteen student interviews supplemented student responses as a deeper probe of misconceptions. In addition to the typical documented misconceptions about gravity, previously undocumented misconceptions were observed. In fact, the breadth of questions allowed a description of possible student mental models. We defined three possible alternative mental models about gravity-the boundary model, the orbital indicator model, and the "mixing of forces model." These alternative models are the first to rigorously generalize student ideas about gravity. Additionally, we explored certain surprising disparate misconceptions and common misapplications of the scientific model in terms of mass, distance, and the cause of gravity. Taken as a whole, our proposed alternative models and description of misapplications of the scientific model offer a new language for astronomy researchers and educators to use in understanding their students' ideas about gravity. These models give educators a vantage point from which to understand their students' background knowledge and to navigate potential pitfalls in learning the scientific model. The results can lead to immediate formative assessment in the classroom and instructional development.

\subsection{Future Plans}

The student-supplied responses and interviews in the present study provide ample information about gravity conceptions in the natural language of students. While information about where these conceptions come from and exactly how students' mental models are structured and used is still in the speculative stage, the project is ongoing and will be developed further in future work. As mentioned earlier, categories that represent misconceptions will be used for distractor choices in an up-and-coming, multiple-choice Newtonian Gravity Concept Inventory (NGCI), which has the potential to provide an independent measure of the mental models presented in Sections 6 and 7. Student interviews will continue to be used to supplement the NGCI results with a qualitative approach in an effort to provide a holistic picture of introductory astronomy student understanding of gravity.

\section{Acknowledgments}

The authors would like to thank the many Physics/Astronomy, Geo-science, and Education experts who provided insight in defining and narrowing the important aspects of gravity. We would also like to thank the Astrolrnr listserv participants who provided anecdotal information about common student misconceptions about gravity, as well as the anonymous reviewer, whose detailed comments helped to refine the paper. And finally, we would like to thank the Montana Space Grant Consortium for providing a graduate fellowship to one of the authors (Williamson) in support of the present research.

\section{Notes}

Note 1: Gregory Francis, Charles Kankelborg, Neil Cornish, Sean Brannon, James Schmidt, Angela Des Jardins.

Note 2: Gregory Francis, Neil Cornish, Charles Kankelborg, Sean Brannon, Angela Des Jardins.

Note 3: James Schmidt

Note 4: David Wittman, Tony George, Karen Castle, Liam McDaid, Chris Sirola, Edward Prather, Nick Strobel, Travis Rector, Lauren Monowar-Jones, Philip Blanco, Ajay Narayanan.

Note 5: Jeffery Adams, Janelle Bailey, Dana Longcope, John Carlsten, John Neumeier 


\section{Appendix A: Questionnaire Results}

Open-response questions posed to students, including the emergent categories, the number of respondents $(\mathrm{N})$ in each category according to both raters, the corresponding percentage of total respondents, and the Cohen's Kappa inter-rater reliability statistic, $\kappa$. The letter before the question number indicates the questionnaire version (A, B, or $\mathrm{C}$ ). The number $\mathrm{N}$ does not include student responses that were placed in different categories according to each rater, nor incoherent or inapplicable student responses. Percentages were calculated by dividing $\mathrm{N}$ by the total number of respondents (143 Version A, 137 for Version B, and 32 for Version C), so percentages may not add up to $100 \%$.

\begin{tabular}{|c|c|c|}
\hline Question Conceptual Categories & $\mathbf{N}$ & $\begin{array}{l}\text { Percent } \\
\text { total }\end{array}$ \\
\hline \multicolumn{3}{|l|}{ A1. Why do astronauts appear to float in their spacecraft ${ }^{\mathrm{i}}$ ? } \\
\hline a. There is no gravity in space. & a. 72 & a. $50.3 \%$ \\
\hline b. Gravity is much weaker in space. & b. 21 & b. $14.6 \%$ \\
\hline c. They are too far away from Earth or any massive body. & c. 15 & c. $10.2 \%$ \\
\hline d. They are in a constant state of freefall. & d. 14 & d. $9.7 \%$ \\
\hline e. The spacecraft's gravity isn't strong enough. & e. 3 & e. $2.1 \%$ \\
\hline
\end{tabular}

A2. If you traveled down a tunnel that reached halfway to the center of the Earth, would the strength of gravity you experience from the Earth change? If so, how? Explain your answer.

a. No change, Earth's gravity is constant and uniform.

b. Increase, you are closer to the center

c. Increase, there is more pressure.

d. Increase, closest to the densest area.

e. Decrease, more mass above you and less beneath you.

f. Increase, closest to the source of gravity.

g. References interaction with magnetic field

h. Discusses influence from other celestial bodies

i. No change, Earth's mass is the same.

j. Increase, smaller radius from center.

$\begin{array}{ll}\text { a. } 33 & \text { a. } 23.1 \% \\ \text { b. } 19 & \text { b. } 13.3 \% \\ \text { c. } 9 & \text { c. } 6.3 \% \\ \text { d. } 7 & \text { d. } 4.9 \% \\ \text { e. } 5 & \text { e. } 3.5 \% \\ \text { f. } 5 & \text { f. } 3.5 \% \\ \text { g. } 4 & \text { g. } 2.8 \% \\ \text { h. } 4 & \text { h. } 2.8 \% \\ \text { i. } 4 & \text { i. } 2.8 \% \\ \text { j. } 3 & \text { j. } 2.1 \%\end{array}$

A3. Is there a relationship between a planet's gravity and its atmosphere? If so, describe this relationship.

a. No, not that they know of.

b. Yes, gravity holds the atmosphere to the planet.

$\begin{array}{ll}\text { a. } 29 & \text { a. } 20.3 \% \\ \text { b. } 23 & \text { b. } 16.1 \% \\ \text { c. } 17 & \text { c. } 11.9 \%\end{array}$

c. Yes, atmosphere affects the strength of gravity.

d. Yes, gravity determines the thickness and composition of the atmosphere.

e. No, gravity depends on mass and distance and can exist without atmosphere.

f. Yes, atmosphere represents the boundary of gravity.

g. Yes, gravity causes the atmosphere to exist.

h. Yes, the atmosphere exerts pressure.

i. Yes, but not sure what relationship.

\section{A4. How heavy or light does something have} to be to create its own gravitational field?

a. Every object has some gravitational field.

b. Very, very heavy.

c. Planet/moon sized.

0.84 
A5. Which person standing on the surface of the Earth experiences a stronger force of gravity? Why?

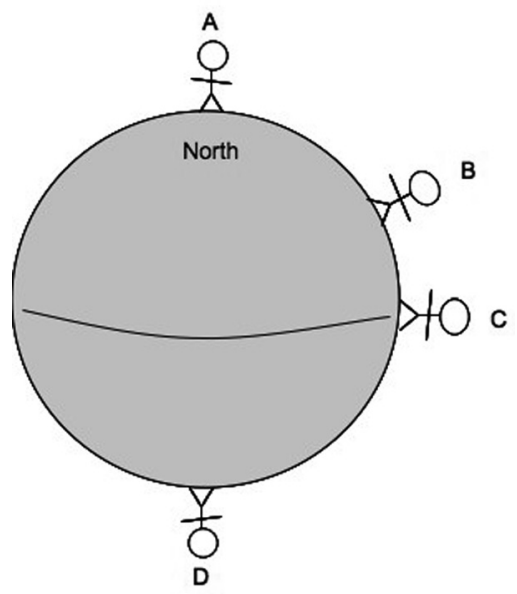

a. Gravity is the same everywhere on the planet.

$\begin{array}{ll}\text { a. } 73 & \text { a. } 51.0 \% \\ \text { b. } 13 & \text { b. } 9.1 \% \\ \text { c. } 8 & \text { c. } 5.6 \% \\ \text { d. } 6 & \text { d. } 4.2 \% \\ \text { e. } 4 & \text { e. } 2.8 \% \\ \text { f. } 4 & \text { f. } 2.8 \% \\ \text { g. } 3 & \text { g. } 2.1 \%\end{array}$

A6. Each of the following planets has the same mass M, but each is made of a different material. The darker the planet, the denser the material. Describe the strength of gravity that a rocket at distance $\mathbf{R}$ from the center of each planet experiences. Why?

।.

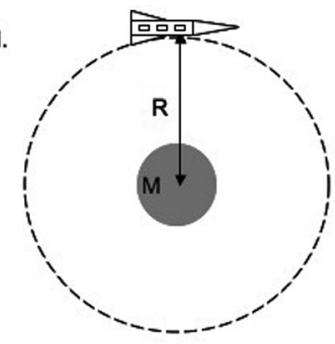

III.

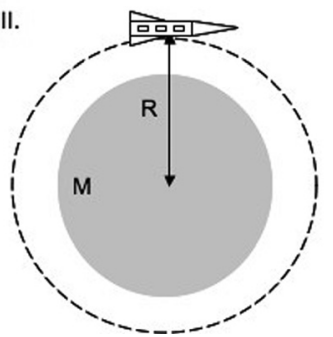

II.
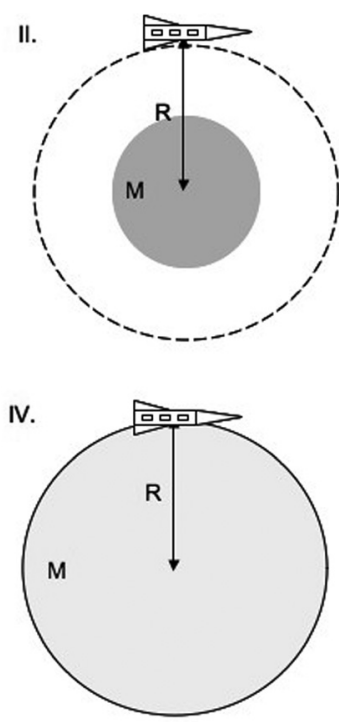
a. Same, equal mass and distance from center.
a. 27
a. $18.9 \%$
b. 18 b. $12.6 \%$

b. Denser material = stronger gravity .

c. Must be "just right" - planet not too dense and rocket not too far (chose B or C).

c. 17 c. $11.9 \%$

d. A is the strongest and D is weakest, no explanation.

d. 15 d. $10.5 \%$

e. Considers distance from the surface of the planet.

f. A is the weakest and D is strongest, no explanation.

g. All same, higher density is compensated by smaller distance to surface.

e. 11 e. $7.7 \%$

f. 8 f. $5.6 \%$

g. 6 g. $4.2 \%$

h. The size of the planet determines gravity.

h. 5 h. $3.5 \%$

\section{A7. Describe the strength of gravity on each of the following planets (if any), where more black loops represent stronger magnetic fields.}
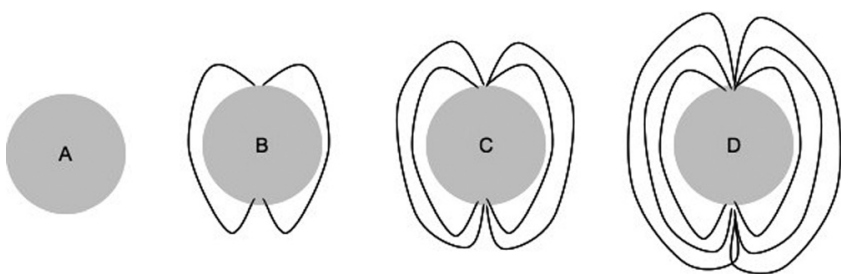

a. More magnetic fields = stronger gravity .
a. 69
a. $48.3 \%$
b. 23 b. $16.1 \%$
c. 11 c. $7.7 \%$
d. 4 d. $2.8 \%$

b. Gravity and magnetism have no relationship.

c. Gravity cannot exist without magnetic fields.

d. Magnetic fields cancel out a planet's gravity (A is strongest).

\section{B8. Why don't the planets fall into the Sun?}
a. Gravity
a. 33
a. $24.1 \%$
b. Orbiting is like falling, centripetal forces.
b. 27
b. $19.7 \%$
c. They are on an established orbit.
c. 13 c. $9.4 \%$
d. Gravity from other bodies in the solar system is pulling them out.
d. 13
d. $9.4 \%$
e. Sun's gravity isn't strong enough to pull them in, only
to hold them in place.
e. 7 e. $5.1 \%$
f. Rotation of planets or the Sun
f. 7 f. $5.1 \%$
g. The planets have their own gravity that counteracts the Sun's.
g. 6
g. $4.4 \%$
h. Sun's magnetic field pushes out.
h. 5 h. $3.6 \%$

0.94

\section{B9. Besides gravity, are there any other forces that hold} us to the Earth's surface?

a. Nothing else.
a. 77 a. $56.2 \%$
b. 17 b. $12.4 \%$
c. 9 c. $6.6 \%$
d. 5 d. $3.6 \%$
e. 4 e. $2.9 \%$
f. 4 f. $2.9 \%$
g. 3 g. $2.2 \%$
h. 2 h. $1.5 \%$

b. Atmospheric forces.

d. Friction.

e. More than one of the above factors

f. Yes, maybe.

g. Magnetic forces.

h. Just provided random Physics terms

B10. The strength of gravity on top of Mt. Everest is $0.25 \%$ less than the strength of gravity at sea level. Why is this?
a. Further from core where gravity is stronger.
a. 39
a. $28.5 \%$
b. Higher elevation, greater distance from center of mass.
b. 32 b. $23.4 \%$ 


\begin{tabular}{l} 
Question Conceptual Categories \\
\hline $\begin{array}{l}\text { c. Higher in atmosphere, closer to space. } \\
\text { d. Less air pressure, air is thinner. }\end{array}$ \\
B11. How would you compare the strength of gravity at the \\
surface of Pluto with the strength of gravity at the surface \\
of the Earth? Explain.
\end{tabular}

a. Weaker, smaller and less mass.

b. Weaker, further from Sun.

c. Need more information, describes how to compare

d. Weaker, no explanation

e. Greater, for various reasons

f. Weaker, Pluto has less atmosphere

g. They're different.

h. The same.
Percent of

total

$\mathbf{K}$

c. 20 c. $14.6 \%$

d. 14 d. $10.2 \%$
0.91
a. 61
a. $44.5 \%$
b. 26 b. $19.0 \%$
c. 9 c. $6.6 \%$
d. 7 d. $5.1 \%$
e. $7 \quad$ e. $5.1 \%$
f. 5 f. $3.6 \%$
g. 4 g. $3.0 \%$
h. 2 h. $1.5 \%$

B12. When the Apollo spacecraft passed behind the moon from our view on Earth, how did the gravity between the Earth and the spacecraft change (if at all)? Explain.

a. No change, gravity can pull through objects.
a. 31
a. $22.6 \%$
b. 12 b. $8.8 \%$
c. 11 c. $8.0 \%$
d. 9 d. $6.6 \%$
e. 5 e. $3.6 \%$

b. Moon's gravity takes over, not feeling Earth's gravity anymore.

d. Now BOTH Earth and Moon are pulling.

B13. The following planets are viewed from above, with more arrows representing a faster rotation. Describe the strength of gravity (if any) on each planet and how it compares to the other planets.
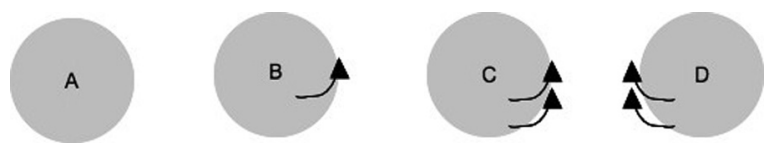

a. More rotation $=$ more gravity .
a. 48
a. $35.0 \%$
b. 29 b. $21.2 \%$
c. 18 c. $13.1 \%$
d. 9 d. $6.6 \%$
e. 9
e. $6.6 \%$

b. All have the same gravity.

c. Gravity is non-existent without rotation (A has no gravity).

e. More rotation = less gravity.

B14. Compare the surface gravity on each of the following planets with different orbiting bodies.

Planet A has nothing orbiting it; B-D have artificial satellites, and E-G have moons. Also, which planets would you expect to have tides?

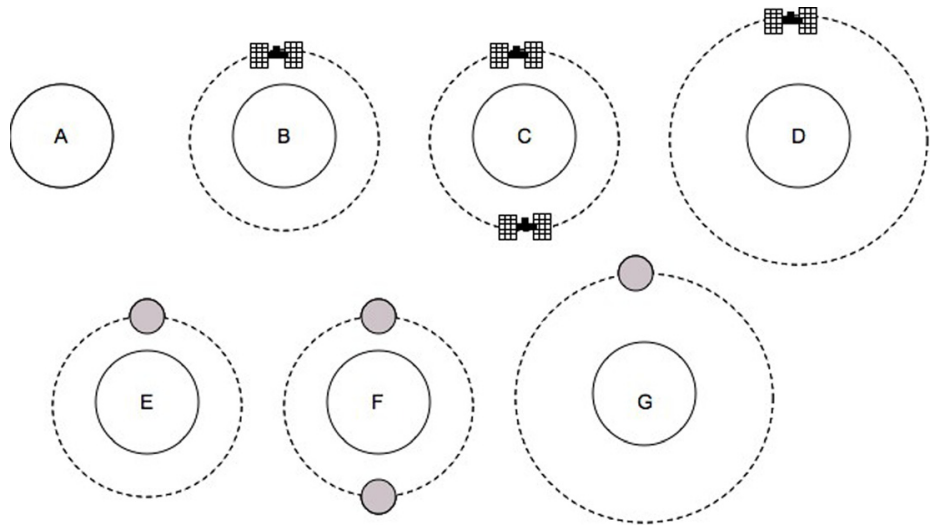


b. Gravity must be strong to hold objects that are far away.
a. 30
a. $21.9 \%$
b. 15 b. $10.9 \%$
c. 14 c. $10.2 \%$

c. Same, surface gravity doesn't depend on what is orbiting.

B15. Compare the strength of the force of gravity for each satellite.

Note that Satellite B is twice as big as Satellite $A$ and

Satellite $\mathrm{C}$ is three times as big as Satellite $\mathrm{A}$.

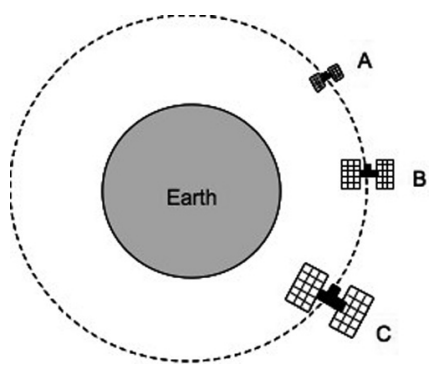

a. Larger, more massive satellites experience a stronger force of gravity.
a. 72
a. $52.6 \%$
b. 35 b. $25.5 \%$
c. 11 c. $8.0 \%$
d. 3 d. $2.2 \%$
e. 2 e. $1.5 \%$

b. Same, no explanation.

c. Smaller satellites experience a stronger pull of gravity

(b/c more easily affected).

d. Same, gravity pulls on all objects equally.

B16. In this diagram, darker planets have a higher density. Which planet would have taller mountains? Why?
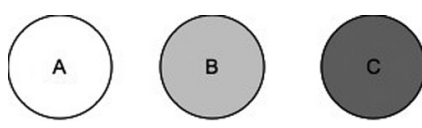
a. A, lower density means more malleable.
a. 33
a. $24.1 \%$
b. B, it's in between, just right
b. 28 b. $20.4 \%$
c. C, more material to make mountains.
c. 22
c. $16.1 \%$
d. A, has less gravity.
d. 15 d. $10.9 \%$
e. C, more geologic activity
e. 7 e. $5.1 \%$
f. Need more information.
f. 6 f. $4.4 \%$

C17. How far away from the Earth do you have to go to feel zero gravitational force from the Earth?
a. Outside of the Earth.
a. 15 a. $46.9 \%$
b. On the scale of much larger than the solar system.
b. 5 b. $15.6 \%$
c. On the scale of nearby bodies and the solar system.
c. 4 c. $12.5 \%$

\section{C18. What determines the strength of the} gravitational interaction between two objects?
a. Mass and/or distance, but incomplete.
a. 16 a. $50.0 \%$
b. Complete: Mass of both objects and distance between them.
b. 11 b. $34.4 \%$
c. Variables other than mass and distance.
c. 2 c. $6.3 \%$ 
C19. How would the motion of a baseball thrown upward from the surface of the Moon differ from that on Earth? Describe how fast it will go and where it will end up.
a. Will land back on surface.
a. 20 a. $62.5 \%$
b. Will travel away from the moon.
b. 4 b. $12.5 \%$
c. Will float.
c. 4 c. $12.5 \%$

0.93

C20. Assume that planets A and B have the same mass M, but planet $A$ is made of a denser material and planet $B$ is bigger. Make an " $X$ " about where a rocket that is launched from planet $A$ begins to experience a stronger force of gravity from planet $B$.

The black dot shows the halfway point for reference.

Explain your choice.

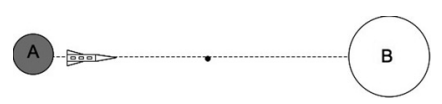
a. Recognizes that pull of gravity would be equal at halfway point
a. 13 a. $40.6 \%$
b. Closer to B, no explanation
b. 8 b. $25.0 \%$
c. B has more gravity because it is larger.
d. A has more gravity because it is more dense.
c. 6 c. $18.8 \%$
e. Closer to A, no explanation
d. 3 d. $9.4 \%$
e. 1 e. $3.1 \%$

C21. Compare the force of gravity at the surface of each of the following planets that have different ring structures.

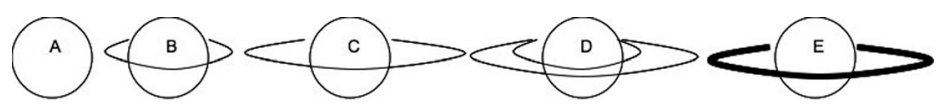

a. More material around a planet $=$ stronger gravity.
a. 13
a. $40.6 \%$
b. 5
b. $15.6 \%$
c. 4
c. $12.5 \%$
d. 2 d. $6.25 \%$

b. Less material around a planet $=$ stronger gravity.

c. Planets with rings far away $=$ more gravity.

d. All the same.

C22. A person is living on a planet where there has been a huge hole carved out. Draw an arrow that shows the direction of the gravitational force he experiences. Explain your choice.

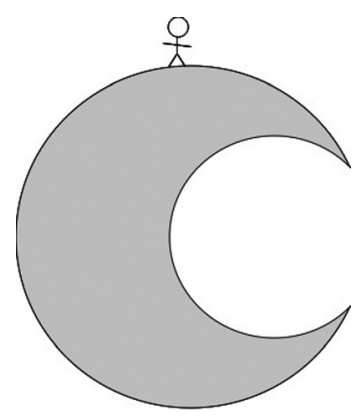
a. Toward new center of mass.
b. Toward geometric center.
c. Toward left surface.
d. Toward the gap.
a. 11
a. $34.4 \%$
b. $7 \quad$ b. $21.9 \%$
c. 4 c. $12.5 \%$
d. 3 d. $9.4 \%$ 
C23. Two astronauts are floating in space far away from any planets or stars. Do they experience any gravitational force?

a. Yes, from each other.

b. Yes, from something else.

c. No, there is no source nearby.

d. No, there is no gravity in space.

e. No, no explanation
a. 12 a. $37.5 \%$
b. 6 b. $18.8 \%$
c. 6 c. $18.8 \%$
d. 3 d. $9.4 \%$
e. 4 e. $12.5 \%$

${ }^{\mathrm{i}}$ Adapted from the TOAST.

${ }^{i i}$ Adapted from Dostal's (2005) Masters Thesis

\section{Appendix B: Interview Results}

Results of student interviews showing each student's major, the questionnaire version he/she was answering, an indicator of his/her consistency in answering questions, description of mental models he/she seemed to display and further notes. To indicate a student's inconsistency in answering questions, a " $\mathrm{C}$ " means that a student appeared confident in his or her responses and consistently applied similar reasoning strategies and an "I" means that a student changed reasoning throughout the interview or could not come to a conclusion on certain questions. Many students were unsure of their answers, with five out of the fifteen interviewees (33\%) ranked as "I", and a few " $C$ " interviews containing notes about the student's lack of confidence.

\begin{tabular}{lccll}
\hline Student and Major & Version & Cor I & Working models \\
\hline 1 University Studies & B & C & Correct scientific model \\
2 Undeclared & B & I & $\begin{array}{l}\text { Boundary model-The Sun is only } \\
\text { strong enough to hold us in place. }\end{array}$
\end{tabular}

$\begin{array}{llll}3 \text { Undeclared } & \text { A } & \text { C } & \begin{array}{l}\text { Boundary model-Different boun- } \\ \text { daries for different masses. The } \\ \text { moon experiences Earth's gravity } \\ \text { but the space station does not. }\end{array} \\ \text { 4 History } & \text { A } \quad \text { C } \quad \begin{array}{l}\text { Boundary model, Misapplication of } \\ \text { Mass: Density }\end{array} \\ 5 \text { Undeclared } & \text { B } & \text { I } & \begin{array}{l}\text { Orbital indicator model } \\ 6\end{array}\end{array}$

6 Undeclared A C Boundary model—Earth's gravity is constant within the atmosphere and then starts diminishing just past the atmosphere.

Orbital indicator-An object's gravity can be measured by its effects on other objects. It is relative; sometimes a body can have a gravitational field if it is the largest Notes

Very unsure of answers, not sure where gravity comes from. Not sure if magnetism and rotation matter.

Very unsure.

There is no gravitational force between Mars and Earth because Mars is not orbiting the Earth. Mars only interacts gravitationally with the Sun.

Magnetic fields and gravity definitely have a relationship; they are not separate. 
7 Health Promotion

8 Architecture (took intro physics)

9 University Studies

10 Business Marketing (took general Physics in high school)
Mixing of forces-The more magnetic fields, the stronger the gravity.

Misapplication of mass: Both mass and density matter, but mostly density.

Boundary model—To a certain extent past the atmosphere, gravity is gone and then you float. If earth did not have an atmosphere, gravity would probably stop inside of Earth. Gravity needs something to permeate through. Mixing of forces-Rotation might affect gravity.

Mixing of forces-The strength of the gravitational force on Mt. Everest is less than at sea level because it is further from the center and from the center of rotation. Orbital indicator-We could look at bodies in the solar system and count how many moons they have and how fast they are orbiting to figure out the planet's surface gravity. Scientific model

Mixing of forces-Planets do not fall into the Sun because their magnetism counteracts the Sun's magnetism. All the heavy metals and iron in Earth's core is pulling us down with magnetism. Also, Venus has a much stronger gravity than Earth b/c its atmosphere is so thick.

I Boundary model—Earth's atmosphere represents where gravity drastically decreases. Also, Earth's gravity is constant in the Earth. When you are flying in a plane nothing feels lighter.

Mixing of forces-Atmosphere causes stronger surface gravity. Also, gravity is determined by a combination of mass and magnetism.

Misapplication of mass_-Earth's mass is the minimum mass for an object to have its own gravitational field. Denser materials have stronger gravity.
Unable to reconcile why planets do not fall into the Sun.

Reasons that planets far away must be more massive for being at such a large distance, but then later reasons that the surface gravity of Jovian planets is less than terrestrial because they are less dense. Very confused about orbiting; it never 'clicked' in class. "Just don't know." Admitted to guessing, sees contradictions, but cannot reconcile them.

Discusses how rotation would change gravity, but when further questioned, admits that gravity is constant and rotation would change the force that is felt.

Claims that most of the answers are just common sense, they just seem right.

There is a complicated interaction between magnetism, rotation, and gravity. There is probably a scientific equation that describes it.

Pluto's surface gravity is much weaker because it is further from the Sun and it is not geologically active.

Unable to reconcile how gravity operates in space.

Earth has gravity because of its mass and it is rotating on axis. Does not understand her own written answers.

Having a gravitational field means being able to affect other objects. Nothing orbits the moon, so it must not create its own gravitational field. Same for Mercury and Venus. Very unsure, guessing at times. Scientists are unable to predict the strength of gravity at the center of the Earth, because they have never been there. 
Student and Major Version Cor I Working models

11 University

Studies

12 University

Studies

B

B

Boundary model-Earth's atmosphere might be able to block outside gravitational forces.

Source of gravity-Originally under the impression gravity comes from something outside Earth, but realized throughout the interview that it comes from a planet.

Mixing of forces-Magnetic fields can counteract gravity. Scientific model-Every object that has mass must have a gravitational field, because it wouldn't make sense for there to be a cut-off mass.

gous to the coin toss machines in
the Mall. The mass of a body deter-
mines the strength of its gravity,
which explains why the Moon and
Pluto have less gravity. The Earth's
gravity comes from its whole mass.
You measure distance from the cen-
ter, but there is nothing magical
about the center that gives it gravity.
Mixing of forces-More rotation
causes less gravity, in analogy to a
merry-go-round.

13 Film

B

14 Psychology planet do not affect it's gravity. Boundary model: The edge of the atmosphere is an indicator of where gravity drastically decreases.

Misapplication of Scientific Model: Gravity is strongest at the center of the Earth. Gravity originates in the core.

C I Boundary model-Gravity dimin-
Notes

Saw inconsistencies in his written answers; rethinks several responses to be more consistent with gravity depending on mass and distance. Uses information about the moon, other planets, and the Sun to try to reason through questions. Wondered if there was really a way to know all the answers with certainty.

Was not sure about the difference between mass and density.

References answers to other questions to reason.

Realizes there is a difference between air pressure and gravity, but each affects the motion of a rocket leaving Earth.

Reasons through questions with ease.

Uses mass and distance consistently as factors that determine gravity. Very sure of answers. Does not question reasoning at all throughout the interview. ishes greatly after atmosphere. Scientific model-Mass and distance determines gravity. The mass of both objects matters: The Earth gravitationally interacts more with the Moon than the Space Station because the Moon is more massive. Misapplication of the Scientific model-Only very massive objects have gravity. Planet do not feel each other's gravity, because they are feeling the Sun's gravity. Empty space (a hole or void) can cause gravity.

Originally says there is no gravity in space, but then discusses how objects in space are affected by gravity in other questions. States that mass and distance determines gravity, but then uses density and physical size of a planet to reason through questions.

Sometimes reasons that objects can only feel gravity from one object at a time, and sometimes discusses how an object can experience gravity from multiple bodies. Changes answer on C23 from correct answer, saying it does not 
Orbital indicator-Rings extend the size of a planet. A planet's physical size determines the extent of its gravitational field.
15 Geology B $\quad$ C Scientific model-A planet's mass alone determines its gravity. Rotation and atmospheric pressure do not affect the strength of gravity. The force of gravity between two bodies depends directly on the mass of both objects. The Earth's gravity is measured from its center because that is the average location of all the mass. make sense now-the astronauts are not massive enough to exert a gravitational force on each other. Objects must be at least the size of a moon to have gravity. Originally reasons that gravity can be determined by apparent weight and its effects on other objects (i.e., up for upward moving objects; a brick buried under the ground would not feel gravity because it cannot move down much farther). However, with more questioning he changes to saying that gravity on Earth is always down.

Gravitational force would pull toward the opening in Question C22 because it would try to seal itself up.

Not entirely confident in the scientific model-thought maybe density could be a factor, but did not include density in reasoning through responses.

Could not figure out why the planets do not fall into the Sun. Thought maybe it had to do with the expansion of space, but not sure.

\section{References}

Agan, L., and Sneider, C. 2004, "Learning about the Earth's Shape and Gravity: A Guide for Teachers and Curriculum Developer," Astronomy Education Review, 2, 90.

Asghar, A., and Libarkin, J. C. 2010, “Gravity, Magnetism and 'Down’: Non-Physics College Students' Conceptions of Gravity," Science Educator, 19, 42.

Bailey, J. 2006, “Development of a Concept Inventory to Assess Students' Understanding and Reasoning Difficulties about the Properties and Formation of Stars," Doctoral Thesis in Teaching and Education, University of Arizona.

Bao, L., and Redish, E. F. 2006, "Model Analysis: Representing and Assessing the Dynamics of Student Learning," Physical Review Special Topics—Physics Education Research, 2, 1.

Bransford, J. D., Brown, A. L., and Cocking, R. R. (eds.), 1999, How People Learn: Brain, Mind, Experience, and School, Washington, DC: National Academy of Sciences.

Chandler, D. 1991, “Weightlessness and Microgravity,” Physics Teacher, 29, 312.

Creswell, J. 2007, Qualitative Inquiry and Research Design, 2nd ed., Thousand Oaks, CA: Sage Publications, Inc.

diSessa, A. 1993, “Toward an Epistemology of Physics,” Cognition and Instruction, 10, 105.

Dostal, J. 2005, “Student Concepts of Gravity,” Masters Thesis in Physics, Iowa State University.

Driver, R. 1985, Children's Ideas in Science, 1st ed., Philadelphia: Open University Press. 
Feeley, R. E. 2007, “Identifying Student Concepts of Gravity,” Masters Thesis in Science and Teaching, The University of Maine.

Fosnot, C. T. (ed.) 1996, Constructivism: Theory, Perspectives, and Practice, New York: Teachers College Press.

Graham, T., and Berry, J. 1993, "Students' Intuitive Understanding of Gravity," International Journal of Mathematical Education in Science and Technology, 243, 473.

Gunstone, R. F., and White, R. T. 1980, “A Matter of Gravity,” Research in Science Education, 10, 35.

Gunstone, R. F., and White, R. T. 1981, "Understanding of Gravity," Science Education, 65, 291.

Hestenes, D., Wells, M., and Swackhamer, G. 1992, “Force Concept Inventory,” The Physics Teacher, $30,141$.

Hobson, A. 2008, “The Surprising Effectiveness of College Scientific Literacy Courses,” The Physics Teacher, $46,404$.

Kavanagh, C., and Sneider, C. 2007a, "Learning About Gravity I. Free Fall: A Guide for Teachers and Curriculum Developers," Astronomy Education Review, 5, 21.

Kavanagh, C., and Sneider, C. 2007b, "Learning About Gravity II. Trajectories and Orbits: A Guide for Teachers and Curriculum Developers," Astronomy Education Review, 5, 53.

Landis, J. R., and Koch, G. G. 1977, “The Measurements of Observer Agreement for Categorical Data," Biometric, 33, 159.

Noce, G. 1988, "The Floating of Objects on the Moon: Prediction From a Theory or Experimental Facts?" International Journal of Science Education, 10, 61.

Nussbaum, J., and Novak, J. 1976, “An Assessment of Children's Concepts of Earth Utilizing Structured Interviews," Science Education, 60, 535.

Nussbaum, J., and Novik, J. 1982, “Alternative Frameworks, Conceptual Conflict and Accommodation: Toward a Principled Teaching Strategy,” Instructional Science, 11, 183.

Palmer, D. 2001, “Students' Alternative Conceptions and scientifically Acceptable Conceptions About Gravity," International Journal of Science Education, 23, 691.

Partridge, P., and Greenstein, G. 2003, "Goals for Astro 101: Report on Workshop for Department Leaders," Astronomy Education Review, 2, 46.

Piburn, M. D., Baker, D. R., and Treagust, D. F. 1988. "Misconceptions About Gravity Held by College Students," Paper Presented at the 61st Annual Meeting of the National Association for Research in Science Teaching, Lake of the Ozarks, M.O.

Prather, E. E., Rudolph, A. L., and Brissenden, G. 2009, “Teaching and Learning Astronomy in the 21st Century," Physics Today, 62, 41.

Redish, E. F. 1994, “The Implications of Cognitive Studies for Teaching Physics," American Journal of Physics, 62, 796.

Sharma, M. D., Millar, R. M., Smith, A., and Sefton, I. M. 2004, “Students' Understanding of Gravity in an Orbiting Space-ship," Research in Science Education, 34, 267.

Slater, T. F., Adams, J. P., Brissenden, G., and Duncan, D. 2001, "What Topics Are Taught in Introductory Astronomy Courses?" The Physics Teacher, 39, 52.

Slater, T. F., and Slater, S. J. 2008, "Development of the Test of Astronomy Standards (TOAST) Assessment Instrument," Bulletin of the American Astronomical Society, 40, 273. 
Smith, C. L., and Treagust, D.F., 1988, "Not Understanding Gravity Limits Students' Comprehension of Astronomy Concepts," The Australian Science Teachers' Journal, 33, 21.

Vosniadou, S. and Brewer, W. F. 1987, “Theories of Knowledge Restructuring in Development," Review of Educational Research, 57, 51.

Watts, D. M. 1982, “Gravity—Don’t Take it for Granted!,” Physics Education, 17, 116.

ÆRR

010105-1-010105-26 